\title{
Investigation of Coulomb dipole polarization effects on reactions involving exotic nuclei
}

\author{
J. P. Fernández-García, ${ }^{1, *}$ M. A. G. Alvarez, ${ }^{2}$ and L. C. Chamon ${ }^{2}$ \\ ${ }^{1}$ INFN, Laboratori Nazionali del Sud, via S. Sofia 62, 1-95123 Catania, Italy \\ ${ }^{2}$ Instituto de Física, Universidade de São Paulo, 05508-090, São Paulo, Brazil \\ (Received 23 March 2015; revised manuscript received 8 May 2015; published 6 July 2015)
}

\begin{abstract}
We have analyzed elastic scattering angular distributions and total reaction cross sections of the exotic nuclei ${ }^{9,11} \mathrm{Li}$ on ${ }^{208} \mathrm{~Pb}$, at energies below and above the Coulomb barrier. For this purpose, we have used an optical potential with no adjustable parameters, composed by the nuclear São Paulo potential, derived from the nonlocal nature of the interaction, and the Coulomb dipole polarization potential, derived from the semiclassical theory of Coulomb excitation. Within this formalism, we identified an unusual long-range absorption for the ${ }^{11} \mathrm{Li}+{ }^{208} \mathrm{~Pb}$ system, which is dominated by the Coulomb interaction. We compare it to the absorption mechanisms observed for ${ }^{6} \mathrm{He}+{ }^{208} \mathrm{~Pb}$ which, unlike those of ${ }^{11} \mathrm{Li}+{ }^{208} \mathrm{~Pb}$, take place at small interacting distances, where both Coulomb and nuclear interactions are important. The proposed approach shows to be a fundamental basis to study reactions involving exotic nuclei.
\end{abstract}

DOI: 10.1103/PhysRevC.92.014604

PACS number(s): 24.10.Ht, 25.60.Bx, 25.60.Dz, 25.70.De

\section{INTRODUCTION}

During most of the 20th century, since the Rutherford experiments [1], we could only perform and analyze nuclear reactions measurements with stable nuclei beams. The recognition of the importance of the exotic nuclei in the stellar environment led to the technological breakthrough of the radioactive ion beam facilities, that make it possible to create and accelerate these rarely found in nature and short-lived nuclei, in laboratory, since 1985 [2]. Then, the most important international laboratories of nuclear physics have invested in research and development for increasing production and improving diagnostics of exotic nuclei beams, thus, providing the study of their structural properties and reaction mechanisms with better statistics.

With precise experimental data of reactions involving exotic nuclei, we can verify whether theoretical models developed to study stable nuclei may be applied to exotic nuclei as well. In such a scenario, the elastic scattering is the simplest process to test a theoretical model, before applying it to more complex reaction processes, and the optical model (OM) is the most used theoretical approach for the corresponding data analysis.

The success of the OM lies in describing reaction mechanisms between two nuclei with a complex mathematical formulation. The mean field potential, called optical potential (OP), has a real part that represents the bare interaction and an imaginary part that simulates the absorption of flux from the elastic channel due to reaction processes [3-5].

Either for older stable nuclei reaction data [3] or recent exotic nuclei ones [4,5], it is commonly assumed the OP with a parametrized Woods-Saxon (WS) form, to represent the strength and geometry of the potentials as a function of the interacting distance.

Recent comparisons between WS-OP analysis of stable and exotic nuclei reactions showed some important differences $[4,5]$. The elastic scattering induced by the ${ }^{6} \mathrm{He}$ exotic nucleus,

*fernandez@lns.infn.it mainly on heavy targets, is different from stable nuclei. A long-range absorption process present in the ${ }^{6} \mathrm{He}+{ }^{208} \mathrm{~Pb}$ scattering data was identified, that can only be reproduced with a significant change in the geometry of the WS-OP, which is achieved by increasing the diffuseness parameter values of such phenomenological (real and imaginary) potentials [4-6]. What was intriguing is that long-range effects were not observed in the light ${ }^{6} \mathrm{He}+{ }^{27} \mathrm{Al}$ or in the heavy ${ }^{9} \mathrm{Li}+{ }^{208} \mathrm{~Pb}$ systems, which are, for example, very well described by similar theoretical models and parameters used for describing stable nuclei reactions $[6,7]$.

It is known that the large number (six) of WS free parameters allows describing a huge number of experimental data in a wide range of bombarding energies, varying from sub-Coulomb to intermediate ones. Notwithstanding, there are difficulties in obtaining simple systematic parameters of the WS potential, with respect to the energy, mass of the system, refractive light effects, or reaction mechanisms, for both stable and exotic systems. Thus, in general, fundamental models for the OP are crucial to describe, in a realistic basis, the energy and system dependency of the data sets.

Stable nuclei reactions have been successfully described assuming the double-folding nuclear São Paulo potential (SPP) [8], among many other theoretical models [9-18]. Within an optical model framework, the SPP describes the real bare nuclear interaction and predicts, with great accuracy, experimental angular distributions of a large number of stable systems in a wide energy range, with no adjustable parameters. At energy regions where the imaginary part of the optical potential can be obtained from fundamental principles, i.e., sub-Coulomb and intermediate energies, predictions of elastic scattering cross sections were obtained in agreement with the experimental data [19-21].

An extension of the SPP model to the imaginary part of the OP was proposed in Ref. [22] and successfully applied to the elastic scattering of stable nuclei. At energies around the Coulomb barrier, the SPP has also been valuable when explicitly coupled to reaction channels [23,24], besides being successful to systematic descriptions of fusion reaction data 
$[25,26]$. Thus, the São Paulo potential became a powerful tool to study exotic nuclei reactions $[6,7,27]$.

The WS-OM analyses performed in Refs. [4-6] showed that the elastic scattering of ${ }^{6} \mathrm{He}$ on ${ }^{208} \mathrm{~Pb}$ is strongly reduced due to the polarization induced by the dipole Coulomb interaction. The so-called Coulomb dipole polarization (CDP) potential accounts for part of this long-range mechanism. A simple analytical formula for the CDP potential was derived in Refs. $[28,29]$ and has shown to be another valuable tool to study exotic nuclei reactions in the OM context [27].

Regarding the study of exotic nuclei, an interesting case consists of the neutron rich weakly bound nuclei, such as ${ }^{6} \mathrm{He}$ and ${ }^{11} \mathrm{Li}$, which are located far away from the stability line of the periodic chart. One goal is to determine, through direct nuclear reactions, how the weakly bound nature of the valence neutrons affects the structure of such exotic nuclei and modifies the way in which they interact.

The ${ }^{11} \mathrm{Li}$ nucleus was first observed in 1966 by Poskanzer et al. [30], through the collision of a $5.3 \mathrm{GeV}$ proton beam on a uranium target; 19 years later, Tanihata et al. [31], proposed, for the first time, a considerably different ${ }^{11} \mathrm{Li}$ nuclear matter radius compared to its neighboring isotopes, which was attributed to the existence of a strong deformation and/or a large extension of its nuclear matter distribution. Two years later, Hansen and Jonson [32] proposed the high probability of finding the weakly bound neutrons far away from the ${ }^{11} \mathrm{Li}$ inner core $\left({ }^{9} \mathrm{Li}\right)$, which makes the ${ }^{11} \mathrm{Li}$ matter density longer and defines it as a nuclear halo. In the same work [32], the existence of a soft dipole resonance was suggested, at low excitation energies, which has been supported by other experimental and theoretical works [33-36].

The ${ }^{11} \mathrm{Li}$ nucleus decays, by $\beta$ emission, in ${ }^{11} \mathrm{Be}$ with a half-life of 8.75(14) ms [37]. It has no excited states and is a Borromean nucleus, since the two subsystems, ${ }^{9} \mathrm{Li}-n$ and $n-n$, are not bound. The energy separation of the two neutrons is $369.15(65) \mathrm{keV}$ [38] and their high probability of being out of the nuclear potential range leads to an extended nuclear halo compared to its isotopes.

The two-neutron halo structure of ${ }^{11} \mathrm{Li}$ is easily polarizable in the strong electric field of a heavy ${ }^{208} \mathrm{~Pb}$ target. The repulsive Coulomb long-range effect forces the ${ }^{9} \mathrm{Li}$ core, with radius $R \sim 2.44$ (7) fm [39], to move in the opposite direction of the two neutrons that do not feel the Coulomb interaction. This effect produces a distortion of the wave function that tends to reduce the Coulomb repulsion between the interacting nuclei. The reduced Coulomb repulsion decreases the elastic scattering cross section and can be described through a dynamic polarization potential, induced by dipole Coulomb excitation. This potential is then composed by two components: an attractive real part that describes the reduction of the Coulomb repulsion and an absorptive imaginary part that describes the elastic cross section reduction $[28,29]$.

As well as ${ }^{11} \mathrm{Li}$, the ${ }^{6} \mathrm{He}$ nucleus presents two weakly bound neutrons halo. The ${ }^{6} \mathrm{He}$ nucleus has a half-life $\tau_{1 / 2}=$ 806.7(1.5) ms [40] and the energy separation of the two neutrons is $972.4(8) \mathrm{keV}$ [41]. The ${ }^{6} \mathrm{He}$ is also a Borromean nucleus, both $n-n$ and ${ }^{4} \mathrm{He}-n$ subsystems are unbound. Similar to ${ }^{11} \mathrm{Li}$, in the case of ${ }^{6} \mathrm{He}$ the positive charge of the ${ }^{4} \mathrm{He}$ core is repelled by the charge of the ${ }^{208} \mathrm{~Pb}$ target. Thus, the Coulomb interaction repels the core ${ }^{4} \mathrm{He}$ in order to separate (break) it from the two neutrons, giving rise to the long-range absorption process.

In this work, we propose to study such long-range absorption of ${ }^{11} \mathrm{Li}+{ }^{208} \mathrm{~Pb}$ through $\mathrm{OM}$ analysis and compare it with the ${ }^{6} \mathrm{He},{ }^{9} \mathrm{Li}+{ }^{208} \mathrm{~Pb}$ ones. In Sec. II, we present our optical model approach. We compare the experimental elastic scattering angular distributions of ${ }^{6} \mathrm{He},{ }^{9} \mathrm{Li}$, and ${ }^{11} \mathrm{Li}$ on ${ }^{208} \mathrm{~Pb}$ with our theoretical predictions in Sec. III. Also in this section, we study the behavior of the total reaction cross sections for these systems. Finally, in Sec. IV we discuss our main results and present the conclusions.

\section{THEORETICAL APPROACH}

The SPP is a microscopic model used to describe the heavy-ion bare interaction [8]. Within this approach, the nuclear interaction, coined as $\mathrm{V}_{\mathrm{SPP}}$, can be written as a function of the folding potential through:

$$
V_{\mathrm{SPP}}(R)=V_{\text {Fold }}(R) \mathrm{e}^{-4 v^{2} / c^{2}},
$$

where $c$ is the speed of light, $v$ is the relative velocity between projectile and target, and $\mathrm{V}_{\text {Fold }}$ is represented as follows:

$$
V_{\mathrm{Fold}}(R)=\iint \rho_{1}\left(\vec{r}_{1}\right) \rho_{2}\left(\vec{r}_{2}\right) V_{0} \delta\left(\vec{R}-\vec{r}_{1}+\vec{r}_{2}\right) d \vec{r}_{1} d \vec{r}_{2} .
$$

$\rho_{1}$ and $\rho_{2}$ are the projectile and target matter distributions; $V_{0} \delta(\vec{r})$ is the zero-range effective interaction with $V_{0}=$ $-456 \mathrm{MeV}$. This $V_{0}$ value has been obtained in [8] through a systematic involving potentials extracted from elastic scattering data analyses. We have used realistic densities for the ${ }^{6} \mathrm{He},{ }^{9} \mathrm{Li}$, and ${ }^{11} \mathrm{Li}$ nuclei extracted from proton scattering measurements $[39,42]$. The corresponding nucleon densities were folded with the matter distribution of the nucleon to obtain the respective matter densities (see Ref. [8]). For the ${ }^{208} \mathrm{~Pb}$ matter density, we assumed the two-parameter Fermi $(2 \mathrm{pF})$ systematic obtained from the São Paulo potential [8]. In Fig. 1(b), we present the ${ }^{6} \mathrm{He}$ matter distribution (solid blue line). As an illustration, we include in the figure the matter distributions of the ${ }^{6} \mathrm{He}$ neighboring isotopes. In Fig. 1(a), we compare the realistic ${ }^{6,9,11} \mathrm{Li}$ matter densities. Different from ${ }^{6,9} \mathrm{Li}$, the ${ }^{11} \mathrm{Li}$ presents a large tail that corresponds to its halo structure.

The CDP potential $[28,29]$ is obtained by requiring that the second-order amplitude for the dipole excitation-de-excitation process and the first-order amplitude, associated with the polarization potential, are equal for all classical trajectories corresponding to a given scattering energy. This leads to an analytic formula for the polarization potential according to a single excited state [28]. This analytic formula is generalized for the case of excitation energy to a continuum of breakup states [29] resulting on the following expression:

$$
\begin{aligned}
U_{\mathrm{Pol}}= & -\frac{4 \pi}{9} \frac{Z_{t}^{2} e^{2}}{\hbar \cdot v} \frac{1}{\left(r-a_{0}\right)^{2} r} \int_{\varepsilon_{b}}^{\infty} d \varepsilon \frac{d B(E 1, \varepsilon)}{\mathrm{d} \varepsilon} \\
& \times\left[g\left(\frac{r}{a_{0}}-1, \xi\right)+i f\left(\frac{r}{a_{0}}-1, \xi\right)\right] .
\end{aligned}
$$




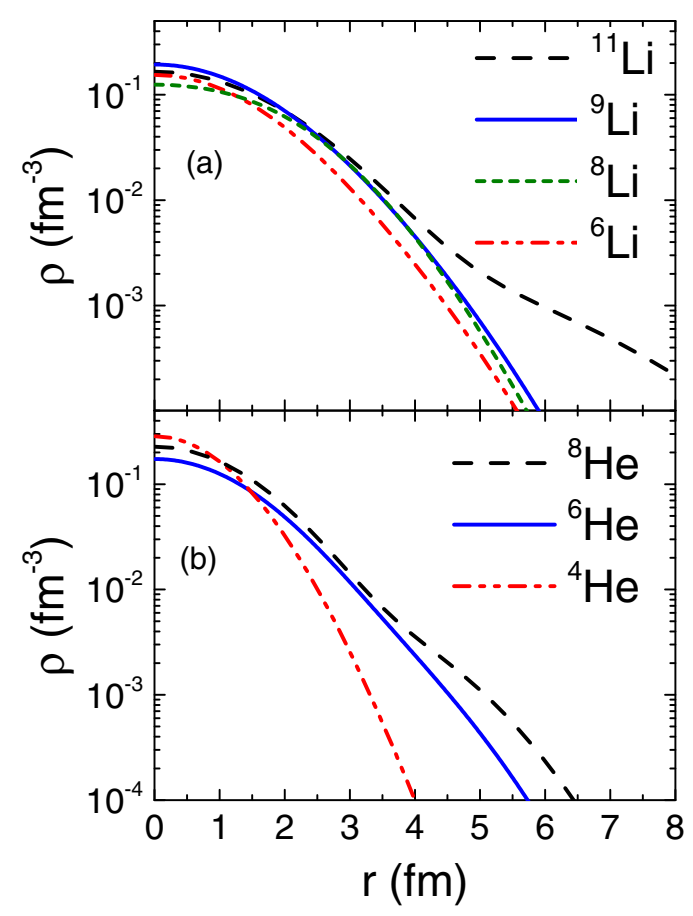

FIG. 1. (Color online) Matter distributions of He and Li isotopes.

Here, we can note the quadratic dependence of the polarization potential with the atomic number of the target, $Z_{\mathrm{t}}$. This dependence gives rise to an important contribution of the Coulomb long-range absorption for certain exotic nuclei, which is mainly observed in the case of the ${ }^{208} \mathrm{~Pb}$ heavy target. In expression (3), $\varepsilon_{b}$ is the necessary energy to break up the projectile, which is a positive value, $a_{0}$ is the half of the distance of closest approach in the head-on collision, $v$ is the velocity of the projectile and $f$ and $g$ are analytic functions expressed as

$$
\begin{aligned}
& f(z, \xi)=4 \xi^{2} z^{2} \exp (-\pi \xi) K_{2 i \xi}^{\prime \prime}(2 \xi z) \\
& g(z, \xi)=\frac{P}{\pi} \int_{-\infty}^{+\infty} \frac{f\left(z, \xi^{\prime}\right)}{\xi-\xi^{\prime}} \mathrm{d} \xi^{\prime} .
\end{aligned}
$$

$\xi=\frac{\varepsilon \cdot a_{0}}{\hbar v}$ is the Coulomb adiabaticity parameter corresponding to the excitation energy $\varepsilon$ of the nucleus. $K^{\prime \prime}$ represents the second derivative of the Bessel functions and $P$ means the principal value of the integral. As discussed in detail in [28], when the breakup energy $\varepsilon_{b}$ is large enough, the purely real adiabatic dipole potential is obtained. In the opposite limit, for small energies, $f\left(\frac{r}{a_{0}}-1, \xi\right) \rightarrow 1$ and $g\left(\frac{r}{a_{0}}-1, \xi\right) \rightarrow 0$, and the polarization potential becomes purely imaginary, depending on $r$ as $\frac{1}{\left(r-a_{0}\right)^{2} r}$.

For the present analysis, we assumed theoretical values of the ${ }^{6} \mathrm{He}$ and ${ }^{11} \mathrm{Li} B(E 1)$ distributions from Refs. [43] and [44], respectively, while for ${ }^{9} \mathrm{Li}$ a cluster model $\left({ }^{7} \mathrm{Li}+2 n\right)$ was considered calculating the $B(E 1)$ distribution $[45,46]$. Figure 2 shows these distributions as functions of the excitation energy $\varepsilon$. The ${ }^{11} \mathrm{Li} B(E 1)$ distribution has also been extracted from measurements of Coulomb dissociation in the collision with ${ }^{208} \mathrm{~Pb}$ [47]. The corresponding results, presented in Fig. 2, are significantly smaller than the theoretical prediction.

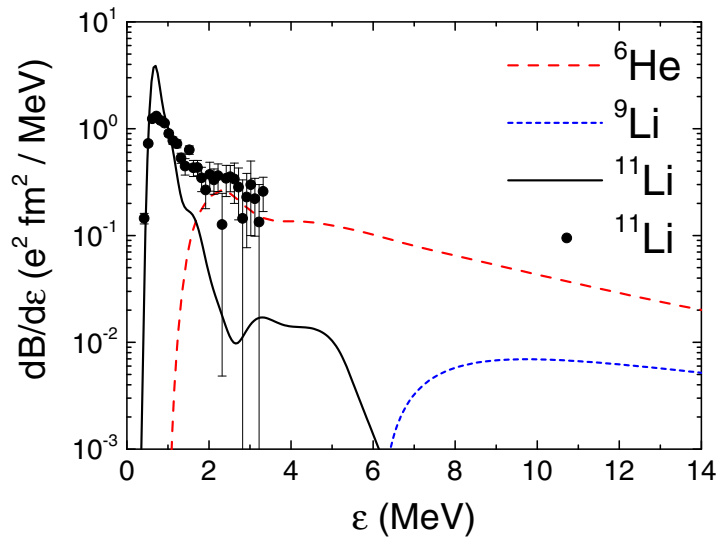

FIG. 2. (Color online) ${ }^{6} \mathrm{He}$ and ${ }^{9,11} \mathrm{Li} B(E 1)$ theoretical distributions as functions of the excitation energy. The figure also presents the ${ }^{11} \mathrm{Li} B(E 1)$ experimental values reported in [47].

Thus, the total optical potential assumed in the present work is written in terms of the SPP and CDP models through

$$
U_{\mathrm{opt}}(R)=V_{\mathrm{SPP}}(R)+i N_{I} V_{\mathrm{SPP}}(R)+V_{\mathrm{Pol}}(R)+i W_{\mathrm{Pol}}(R) .
$$

In [22], the average value $N_{I}=0.78$ was obtained from elastic scattering data analyses performed for several systems involving stable nuclei. In the present work, we have assumed such average value. $V_{\mathrm{Pol}}$ and $W_{\mathrm{Pol}}$ represent the real and imaginary components of $U_{\mathrm{Pol}}$, according to Eq. (3). Thus

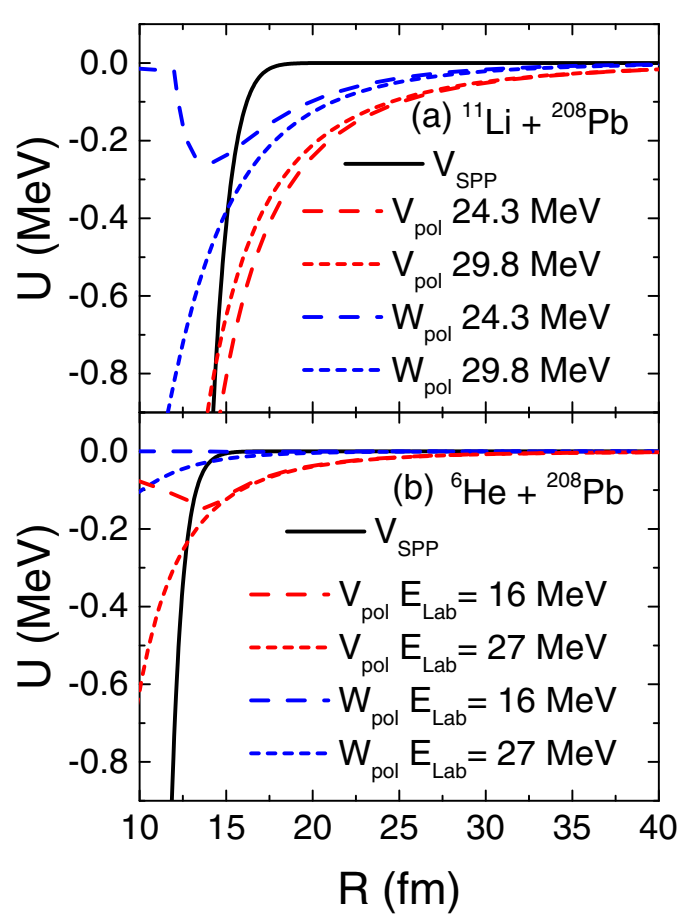

FIG. 3. (Color online) Different components of the total optical potential for ${ }^{6} \mathrm{He},{ }^{11} \mathrm{Li}+{ }^{208} \mathrm{~Pb}$ at bombarding energies around the Coulomb barrier. 
Eq. (5) does not involve any adjustable parameter to fit the present data set.

Figure 3 shows the strength of each component of the OP from Eq. (5), as a function of the interacting distance, for two different incident energies. For both ${ }^{6} \mathrm{He}+{ }^{208} \mathrm{~Pb}$ and ${ }^{11} \mathrm{Li}+{ }^{208} \mathrm{~Pb}$ systems, the strength of the real part of the CDP is significant even well above $R=20 \mathrm{fm}$, while the SPP vanishes exponentially around $14 \mathrm{fm}$. Also the imaginary part of the CDP is significant at large $R$ values for ${ }^{11} \mathrm{Li}$. On the other hand, for ${ }^{6} \mathrm{He}+{ }^{208} \mathrm{~Pb}$ it tends to vanish at long distances.

\section{EXPERIMENTAL DATA AND THEORETICAL PREDICTIONS}

Elastic scattering angular distributions for ${ }^{9} \mathrm{Li}$ on ${ }^{208} \mathrm{~Pb}$ at $E_{\text {lab }}=24.1$ and $29.5 \mathrm{MeV}$ and for ${ }^{11} \mathrm{Li}$ on ${ }^{208} \mathrm{~Pb}$ at $E_{\mathrm{lab}}=$ 24.3 and $29.8 \mathrm{MeV}$ were measured at the TRI-laboratory University Meson Facility (TRIUMF), in Vancouver, Canada, and published in Ref. [7]. Elastic scattering angular distributions of ${ }^{6} \mathrm{He}$ on ${ }^{208} \mathrm{~Pb}$ at $E_{\text {lab }}=14,16,18,22$, and $27 \mathrm{MeV}$ were measured in the Cyclotron Research Center (CRC), Louvain la Neuve, Belgium, and published in $[4,48,49]$. Figures 4, 5, 6, and 7 present these experimental data. The solid black lines in the figures represent the theoretical elastic scattering angular distributions obtained with the total OP given by Eq. (5).

For the ${ }^{9} \mathrm{Li}+{ }^{208} \mathrm{~Pb}$ system, the elastic cross sections obtained with and without considering the CDP potential are very similar and indistinguishable in the scale of Fig. 4. The theoretical results are in quite good agreement with the data. Therefore, even in this case involving an exotic nucleus,

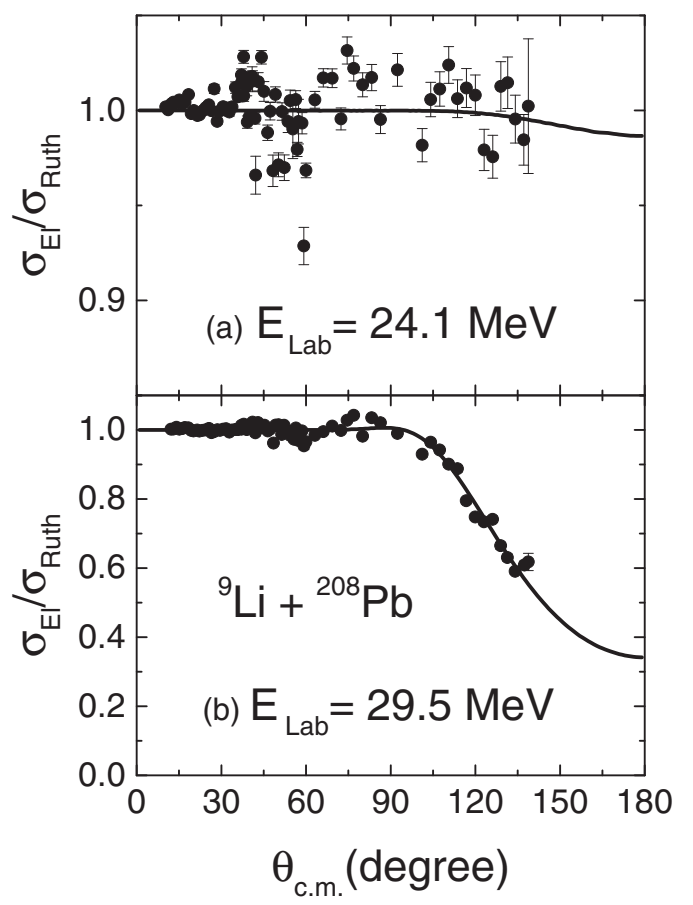

FIG. 4. Elastic scattering angular distributions for ${ }^{9} \mathrm{Li}+{ }^{208} \mathrm{~Pb}$ at 24.1 and $29.5 \mathrm{MeV}$ (data from [7]). The lines represent the theoretical predictions obtained considering the OP of Eq. (5).

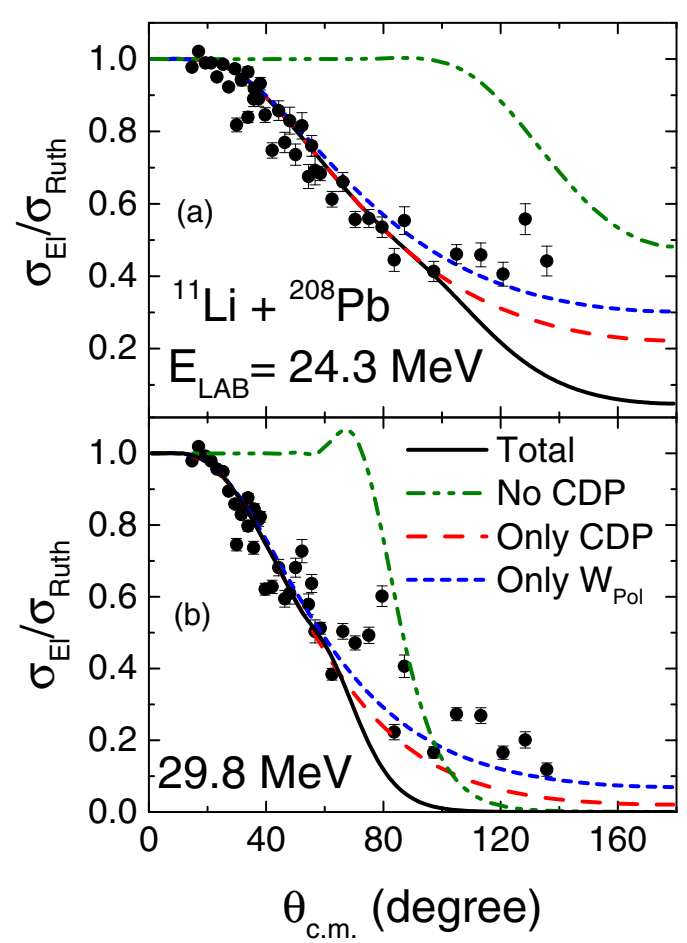

FIG. 5. (Color online) Elastic scattering angular distributions for ${ }^{11} \mathrm{Li}+{ }^{208} \mathrm{~Pb}$ (data from [7]). The lines represent theoretical results obtained considering different optical potential components (see text for details).

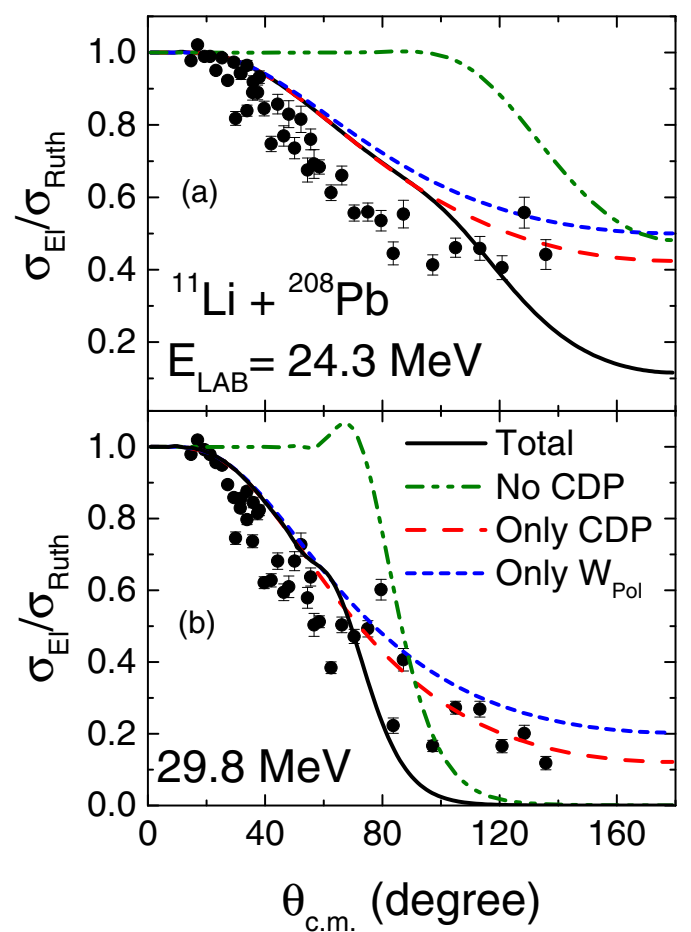

FIG. 6. (Color online) The same of Fig. 5, but assuming in the calculations the ${ }^{11} \mathrm{Li} B(E 1)$ experimental distribution reported in [47]. 

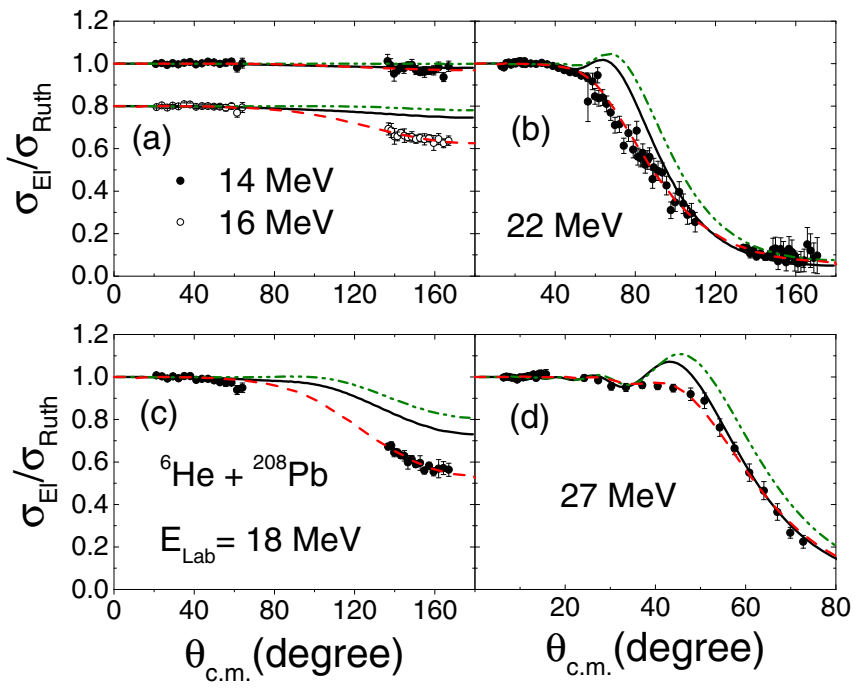

FIG. 7. (Color online) Elastic scattering angular distributions for ${ }^{6} \mathrm{He}+{ }^{208} \mathrm{~Pb}$ from $[4,48,49]$. The elastic cross sections for $E_{\mathrm{lab}}=$ $16 \mathrm{MeV}$ are displaced by a constant value (0.2) to avoid superposition with the data of $E_{\text {lab }}=14 \mathrm{MeV}$. The solid black lines represent the results obtained with the total optical potential of Eq. (5), while the dashed-dot-dot green lines are the results without the CDP potential. The dashed red lines correspond to the theoretical cross sections of Ref. [27].

the CDP is negligible and the total optical potential can be approximated to $U_{\mathrm{opt}} \approx V_{\mathrm{SPP}}+i 0.78 V_{\mathrm{SPP}}$.

In Fig. 5, the solid black lines represent the theoretical elastic scattering angular distributions obtained with the total OP, while the dot-dashed green ones represent the results obtained without the CDP, i.e., with $U_{\mathrm{opt}}=V_{\mathrm{SPP}}+i N_{I} V_{\mathrm{SPP}}$. Thus, unlike the ${ }^{9} \mathrm{Li}+{ }^{208} \mathrm{~Pb}$ case, the elastic cross sections for ${ }^{11} \mathrm{Li}+{ }^{208} \mathrm{~Pb}$ are quite sensitive to the long-range polarization potential. Still in Fig. 5, for comparison purposes, the dashed lines represent the calculations considering only the CDP potential, $U_{\text {opt }}(R)=V_{\text {Pol }}(R)+i W_{\text {Pol }}(R)$, and the dotted lines correspond to the calculations considering only the imaginary part of the CDP, $U_{\mathrm{opt}}(R)=i W_{\mathrm{Pol}}(R)$. It is quite interesting that the cross sections at forward angles are mostly determined just by the long-range Coulomb absorption. The good agreement between data and theoretical calculations in this region is an indication of the Coulomb dipole polarizability dominance, successfully described by the CDP model [28,29].

As mentioned earlier, the experimental ${ }^{11} \mathrm{Li} B(E 1)$ distribution reported in [47] presents significantly smaller values than the theoretical one of [44]. In order to test the effect of this difference on the elastic scattering cross sections, we have also performed OM calculations assuming such experimental distribution, by replacing the $B(E 1)$ in Eq. (3) by a function fitting the experimental data shown in Fig. 2. The dominance of the CDP at forward angles remains in this case, presented in Fig. 6, but the corresponding results are not in good agreement with the data as those from the theoretical $B(E 1)$ distribution.

In Fig. 7, we present ${ }^{6} \mathrm{He}+{ }^{208} \mathrm{~Pb}$ experimental data and corresponding theoretical results obtained with the total OP (solid black lines). The elastic cross sections for $E_{\text {lab }}=16 \mathrm{MeV}$ are displaced by a constant value (0.2) to avoid superposition with the data of $E_{\text {lab }}=14 \mathrm{MeV}$. Also the cross sections obtained without the CDP potential are represented by dot-dot-dashed green lines in the figure. The effect of the long-range polarization is significantly smaller in this case in comparison with that of ${ }^{11} \mathrm{Li}+{ }^{208} \mathrm{~Pb}$. Even so, the CDP is responsible for an improvement in the description of the data also for ${ }^{6} \mathrm{He}+{ }^{208} \mathrm{~Pb}$ (see Fig. 7). Nevertheless, clearly the agreement between data and theoretical predictions with the total OP is not totally satisfactory. In fact, for the ${ }^{6} \mathrm{He}$ system, the $B(E 1)$ is significantly smaller than that obtained for the

${ }^{11} \mathrm{Li}$ case, in the region of the breakup threshold (see Fig. 2). Besides, in the ${ }^{6} \mathrm{He}$ case, other couplings to the $1 n$ and $2 n$ transfer channels and the nuclear breakup are also important, as discussed in Ref. [6].

As already mentioned, the ${ }^{11} \mathrm{Li}+{ }^{208} \mathrm{~Pb}$ elastic scattering cross sections are quite unresponsive to the nuclear part of the optical potential, except at backward angles (see Fig. 5). This fact can be understood by analyzing the strengths of the optical potential components (see Fig. 3). The CDP potential components are of much longer range compared to the São Paulo nuclear interaction, even considering the realistic ${ }^{11} \mathrm{Li}$ nuclear matter distribution represented in Fig. 1. To illustrate this behavior, we have performed additional calculations of reaction cross sections as discussed below.

The reaction cross section is related to the elastic $S$ matrix through the sum of partial waves as

$$
\sigma=\sum_{J} \sigma_{J}=\frac{\pi}{k^{2}} \sum_{J}(2 J+1)\left(1-\left|S_{J}\right|^{2}\right) .
$$

On the other hand, the partial reaction cross section is related to the imaginary part of the OP and the elastic wave function from $[12,50]$ :

$$
\sigma_{J}=-(2 J+1) \frac{4 \pi}{k E} \int W(R)\left|F_{J}(R)\right|^{2} d R .
$$

We now define the reaction cross section density (RCSD) as

$$
\frac{d \sigma}{d R}(R)=-\sum_{J}(2 J+1) \frac{4 \pi}{k E} W(R)\left|F_{J}(R)\right|^{2} .
$$

With Eq. (8), the reaction cross section can be obtained by integration of the RCSD in the interacting distance:

$$
\sigma=\int \frac{d \sigma}{d R} d R
$$

Figures 8(a) and 9(a) present the RCSD as a function of the interacting distance for ${ }^{11} \mathrm{Li}+{ }^{208} \mathrm{~Pb}$ at $E_{\text {lab }}=24.3$ and $29.8 \mathrm{MeV}$, respectively. Here, we separate the total absorption of Eq. (5) in two contributions: the short-range one calculated using the internal imaginary nuclear potential, $W_{\text {SPP }}=N_{I} V_{\text {SPP }}$, and the long-range CDP imaginary part $W_{\text {Pol }}$. Figures 8(b) and 9(b) present the corresponding partial-waves contributions as a function of the angular momentum. Clearly, the absorption due to the CDP is much larger than that arising from the internal $W_{\text {SPP }}$. High angular momenta provide significant contributions to the reaction even in this low energy range. Furthermore, the absorption occurs predominantly at distances much larger than the barrier radius [arrows in Figs. 8(a) and 9(a)]. Similar analyses were performed for 

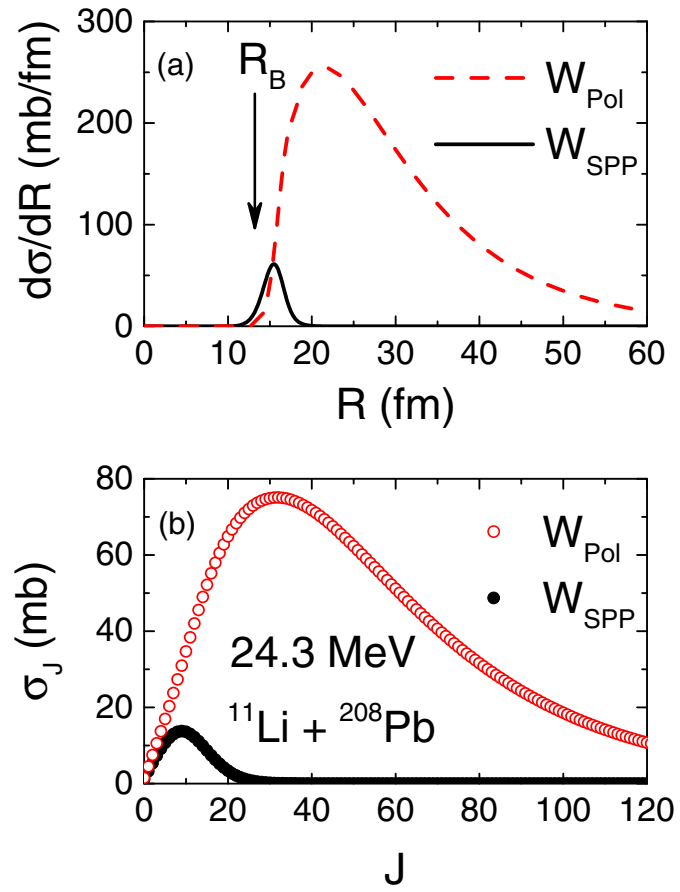

FIG. 8. (Color online) (a) Reaction cross section density as a function of the interacting distance and (b) partial reaction cross section as a function of the angular momentum, for the ${ }^{11} \mathrm{Li}+{ }^{208} \mathrm{~Pb}$ system at $E_{\text {lab }}=24.3 \mathrm{MeV}$. The figure shows the contributions of the inner $\left(W_{\mathrm{SPP}}\right)$ and long-range $\mathrm{CDP}\left(W_{\mathrm{Pol}}\right)$ imaginary potentials. The arrow indicates the approximate position of the $s$-wave barrier radius.
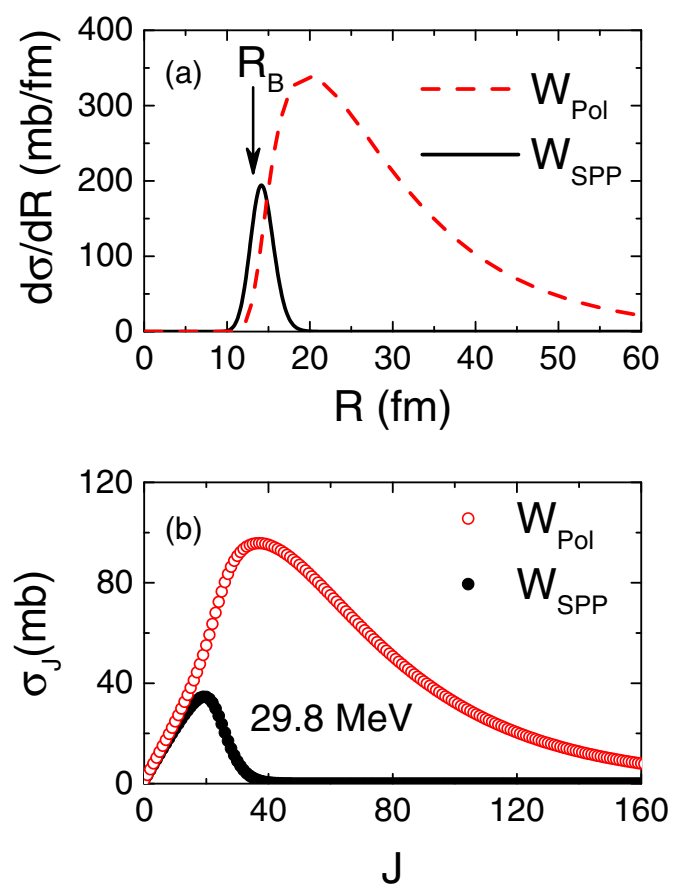

FIG. 9. (Color online) The same as Fig. 8 for $E_{\text {lab }}=29.8 \mathrm{MeV}$. the ${ }^{6,7} \mathrm{Li}+{ }^{80} \mathrm{Se}$ systems, where the absorption occurs at much shorter range $(\approx 10 \mathrm{fm}$ - see Fig. 8 of Ref. [51]). The present results confirm the major role played by the long-range Coulomb absorption in the case of ${ }^{11} \mathrm{Li}+{ }^{208} \mathrm{~Pb}$, and are consistent with the behavior of the elastic scattering cross sections shown in Fig. 5.

\section{DISCUSSION AND CONCLUSIONS}

We have performed OM analyses assuming the OP given by Eq. (5). Within this context, we revisited the case of ${ }^{6} \mathrm{He}+{ }^{208} \mathrm{~Pb}$. The predictions of our OM calculations do not reproduce the corresponding data possibly due to important contributions of the $1 n$ and $2 n$ transfer channels and the nuclear breakup that are not explicitly accounted for our model. In Ref. [27], given the impossibility to reproduce the ${ }^{6} \mathrm{He}+{ }^{208} \mathrm{~Pb}$ data, mainly above the barrier, it was proposed the following construction for the optical potential:

$U_{\mathrm{opt}}(R)=V_{\mathrm{SPP}}(R)+U_{\mathrm{Pol}}(R)+i W_{S}(R)+V_{L}(R)+W_{L}(R)$,

where $W_{S}(R)$ and $W_{L}(R)$ represent short- and long-range components of the phenomenological imaginary nuclear polarization potential and $V_{L}(R)$ is a real nuclear part of such polarization. This construction provided good fits of the ${ }^{6} \mathrm{He}+{ }^{208} \mathrm{~Pb}$ data (see dashed red lines in Fig. 7) and led to more complex CRC calculations [6].

In Ref. [6], both the elastic scattering and the $\alpha$ production, by breakup, were studied for ${ }^{6} \mathrm{He}+{ }^{208} \mathrm{~Pb}$. The experimental data set was compared to results of continuum discretized coupled channels and coupled reaction channels (CRC) calculations. In the CRC case, the coupling of two neutrons transfer channels produces a strong effect on the elastic scattering cross sections, and it better explains the energy and angular distributions of the $\alpha$ particles. In these calculations, the SPP was also assumed for the bare interaction, supplemented with a short-range imaginary potential to account for complete fusion. Although there is room for improvement, these calculations indicate a direction for new theoretical developments.

The predictions of the present OM calculations for the elastic scattering cross sections are in good agreement with the ${ }^{9,11} \mathrm{Li}+{ }^{208} \mathrm{~Pb}$ data (see Figs. 4 and 5). In the case of ${ }^{9} \mathrm{Li}$, the calculations present the major role played by the nuclear potential in the corresponding elastic scattering angular distribution. On the other hand, our analyses demonstrate the dominance of a Coulomb dipole potential in the description of the elastic cross sections for ${ }^{11} \mathrm{Li}+{ }^{208} \mathrm{~Pb}$. This effect is mainly responsible for the unusual long-range absorption observed in this case and it is well accounted for the CDP potential. The respective attractive real part describes the reduction of the Coulomb repulsion and the absorptive imaginary part results in the pronounced damping of the elastic cross section. This behavior is corroborated by the calculated RCSD, which shows that the absorption mostly takes place in the region of large interaction distances.

As illustrated in Fig. 5, a better data description, mainly at the backward region, seems to be obtained by considering only the CDP, Eq. (3), in Eq. (5) instead of the complete interaction. 
However, due to statistical fluctuations and precision of the experimental data at such region, further conclusions must be taken with care.

As already commented, in the case of ${ }^{11} \mathrm{Li}+{ }^{208} \mathrm{~Pb}$ the theoretical $B(E 1)$ distribution reported in [44] provides better description of the elastic scattering data set than the experimental one of [47]. We point out that the theoretical $B(E 1)$ distribution also provides good data description for the breakup probabilities angular distributions [44]. The increasing of the $B(E 1)$ distribution, at low excitation energies, is characteristic of halo nuclei, due to their normally low binding energies. In the specific case of ${ }^{11} \mathrm{Li}$, the increasing of the $B(E 1)$ distribution can also be due to the presence of a dipolar resonance at a low excitation energy. In [44], the experimental breakup cross sections of ${ }^{11} \mathrm{Li}$ on ${ }^{208} \mathrm{~Pb}$ are described by assuming a resonance with energy of $0.69 \mathrm{MeV}$ (which is $0.32 \mathrm{MeV}$ above the breakup threshold). Other theoretical models predict the existence of such low-lying dipole resonance [34-36].

Thus, we were able to study reactions involving different exotic nuclei consistently within the same model. Our OP approach should be a fundamental basis to study other reactions involving exotic nuclei.

\section{ACKNOWLEDGMENTS}

The authors thank Dr. A. M. Moro for his fruitful comments. This work was partially supported by the Fundação de Amparo à Pesquisa do Estado de São Paulo (FAPESP), Brazil, the Conselho Nacional de Desenvolvimento Científico e Tecnológicoo (CNPq), Brazil, and the Istituto Nazionale di Fisica Nucleare (INFN), Italy.
[1] E. Rutherford, Philos. Mag. 37, 581 (1919).

[2] I. Tanihata et al., Phys. Lett. B 160, 380 (1985).

[3] J. Cook et al., Nucl. Phys. A 388, 173 (1982).

[4] A. M. Sánchez-Benítez et al., Nucl. Phys. A 803, 30 (2008).

[5] O. R. Kakuee et al., Nucl. Phys. A 765, 294 (2006).

[6] J. P. Fernández-García, M. A. G. Alvarez, A. M. Moro, and M. Rodríguez-Gallardo, Phys. Lett. B 693, 310 (2010).

[7] M. Cubero et al., Phys. Rev. Lett. 109, 262701 (2012).

[8] L. C. Chamon, B. V. Carlson, L. R. Gasques, D. Pereira, C. De Conti, M. A. G. Alvarez, M. S. Hussein, M. A. Cândido Ribeiro, E. S. Rossi, and C. P. Silva, Phys. Rev. C 66, 014610 (2002).

[9] G. R. Satchler and W. G. Love, Phys. Rep. 55, 183 (1979).

[10] G. R. Satchler, Direct Nuclear Reactions (Clarendon Press, Oxford, 1983).

[11] G. R. Satchler, Phys. Rep. 199, 147 (1991).

[12] G. R. Satchler, M. A. Nagarajan, and I. J. T. J. S. Lilley, Ann. Phys. (NY) 178, 110 (1987).

[13] D. T. Khoa, Nucl. Phys. A 484, 376 (1988).

[14] M. E. Brandan and G. R. Satchler, Nucl. Phys. A 487, 477 (1988).

[15] S. M. Lenzi, A. Vitturi, and F. Zardi, Phys. Rev. C 40, 2114 (1989).

[16] G. R. Satchler, Nucl. Phys. A 579, 241 (1994).

[17] M. E. Brandan, H. Chehime, and K. W. McVoy, Phys. Rev. C 55, 1353 (1997).

[18] M. E. Brandan and G. R. Satchler, Phys. Rep. 285, 143 (1997).

[19] M. A. Cândido Ribeiro, L. C. Chamon, D. Pereira, M. S. Hussein, and D. Galetti, Phys. Rev. Lett. 78, 3270 (1997).

[20] L. C. Chamon, D. Pereira, M. S. Hussein, M. A. Cândido Ribeiro, and D. Galetti, Phys. Rev. Lett. 79, 5218 (1997).

[21] L. C. Chamon, D. Pereira, and M. S. Hussein, Phys. Rev. C 58, 576 (1998).

[22] M. A. G. Alvarez, L. C. Chamon, M. S. Hussein, D. Pereira, L. R. Gasques, E. S. Rossi, and C. P. Silva, Nucl. Phys. A 723, 93 (2003).

[23] D. Pereira et al., Phys. Rev. C 74, 034608 (2006).

[24] L. F. Canto et al., Nucl. Phys. A 821, 51 (2009).

[25] L. R. Gasques, L. C. Chamon, D. Pereira, M. A. G. Alvarez, E. S. Rossi, C. P. Silva, and B. V. Carlson, Phys. Rev. C 69, 034603 (2004).

[26] G. P. A. Nobre, L. C. Chamon, L. R. Gasques, B. V. Carlson, and I. J. Thompson, Phys. Rev. C 75, 044606 (2007).
[27] J. P. Fernández-García, M. Rodríguez-Gallardo, M. A. G. Alvarez, and A. M. Moro, Nucl. Phys. A 840, 19 (2010).

[28] M. V. Andrés, J. Gómez-Camacho, and M. A. Nagarajan, Nucl. Phys. A 579, 273 (1994).

[29] M. V. Andrés, J. Gómez-Camacho, and M. A. Nagarajan, Nucl. Phys. A 583, 817 (1995).

[30] A. M. Poskanzer, S. W. Cosper, E. K. Hyde, and J. Cerny, Phys. Rev. Lett. 17, 1271 (1966).

[31] I. Tanihata et al., Phys. Rev. Lett. 55, 2676 (1985).

[32] P. G. Hansen and B. Jonson, Euro. Phys. Lett. 4, 409 (1987).

[33] A. A. Korsheninnikov et al., Phys. Rev. Lett. 78, 2317 (1997).

[34] K. Ikeda, Nucl. Phys. A 538, 355 (1992).

[35] E. Garrido, D. V. Fedorov, and A. S. Jensen, Nucl. Phys. A 708, 277 (2002).

[36] E. C. Pinilla, P. Descouvemont, and D. Baye, Phys. Rev. C 85, 054610 (2012).

[37] J. H. Kelley, E. Kwan, J. E. Purcell, C. G. Sheu, and H. R. Weller, Nucl. Phys. A 880, 88 (2012).

[38] M. Smith et al., Phys. Rev. Lett. 101, 202501 (2008).

[39] A. V. Dobrovolsky et al., Nucl. Phys. A 766, 1 (2006).

[40] D. R. Tilley, C. M. Cheves, J. L. Godwin, G. M. Hale, H. M. Hofmann, J. H. Kelley, C. G. Sheu, and H. R. Weller, Nucl. Phys. A 708, 3 (2002).

[41] G. Audi, A. H. Wapstra, and C. Thibault, Nucl. Phys. A 729, 337 (2003).

[42] G. D. Alkhazov et al., Nucl. Phys. A 712, 269 (2002).

[43] M. Rodríguez-Gallardo, J. M. Arias, J. Gómez-Camacho, A. M. Moro, I. J. Thompson, and J. A. Tostevin, Phys. Rev. C 80, 051601(R) (2009).

[44] J. P. Fernández-García et al., Phys. Rev. Lett. 110, 142701 (2013).

[45] Y. Alhassid, M. Gai, and G. F. Bertsch, Phys. Rev. Lett. 49, 1482 (1982)

[46] C. A. Bertulani, G. Baur, and M. S. Hussein, Nucl. Phys. A 526, 751 (1991).

[47] T. Nakamura et al., Phys. Rev. Lett. 96, 252502 (2006).

[48] O. R. Kakuee et al., Nucl. Phys. A 728, 339 (2003).

[49] L. Acosta et al., Phys. Rev. C 84, 044604 (2011).

[50] L. C. Chamon, L. R. Gasques, L. F. M. Alves, V. Guimarães, P. Descouvemont, R. J. deBoer, and M. Wiescher, J. Phys. G: Nucl. Part. Phys. 41, 035101 (2014).

[51] L. Fimiani et al., Phys. Rev. C 86, 044607 (2012). 